\title{
Automated Segmentation of Iris Images Using Visible Wavelength Face Images
}

\author{
Chun-Wei Tan, Ajay Kumar \\ Department of Computing, The Hong Kong Polytechnic University, Hung Hom, Hong Kong \\ cscwtandcomp.polyu.edu.hk, a jaykraieee.org
}

\begin{abstract}
Remote human identification using iris biometrics requires the development of automated algorithm of the robust segmentation of iris region pixels from visible face images. This paper presents a new automated iris segmentation framework for iris images acquired at-a-distance using visible imaging. The proposed approach achieves the segmentation of iris region pixels in two stages, i.e. (i) iris and sclera classification, and (ii) post-classification processing. Unlike the traditional edge-based segmentation approaches, the proposed approach simultaneously exploits the discriminative color features and localized Zernike moments to perform pixel-based classification. Rigorous experimental results presented in this paper confirm the usefulness of the proposed approach and achieve improvement of $42.4 \%$ in the average segmentation errors, on UBIRIS.v2 dataset, as compared to the previous approach.
\end{abstract}

\section{Introduction}

Iris recognition has been emerge as one of the most preferred biometric modalities for automated personal identification. Conventional iris recognition systems have been designed to work in strictly constrained environments in order to mitigate the influence of the noises from various sources such as illumination changes, occlusions from eyeglasses, eyelashes, hair and reflections, just to name a few. The systems are usually operate in near-infrared (NIR) illumination with wavelengths in between $700-900 \mathrm{~nm}$, and require the subjects to provide images from short distance of $1-3 \mathrm{ft}$ $[1,2]$. The use of NIR illumination devices requires extra precautions and safety measurements as human eyes are not instinctively responsive to the NIR illumination. Excessive level of NIR illumination can cause permanent damage to the human eyes $[3,4,5]$.

There have been recent efforts to acquire iris images using visible illumination (visible wavelength, $\mathrm{VW}$ ), to overcome limitations of current iris recognition systems and develop less cooperative iris recognition for higher security and surveillance. Acquisition using VW is less hazardous

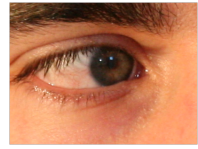

(a)

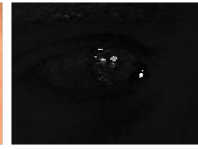

(b)

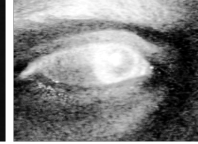

(c)

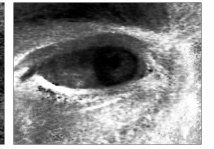

(d)
Figure 1. Sclera feature (a) source image, (b) hue, (c) chroma blue, (d) chroma red.

to human eyes as compared to NIR illumination and is suitable to work in unconstrained environments. Subjects are no longer required to conform to the stop-and-stare mode in the unconstrained environments. Reference [3, 6] are the typical examples of such visible illumination acquired databases which are publicly available. In addition, the advanced imaging technologies, for example, high resolution CMOS/CCD cameras, are now available to conveniently acquire high resolution images at distances beyond $3 \mathrm{ft}$ using visible illumination and locate iris images suitable for recognition.

Integro-differential operator or its derivatives have shown effectiveness on those iris images acquired in controlled environments using NIR illumination $[2,7,8,9,10]$, where there is a significant contrast between pupilary and limbic boundaries. However, these conventional edgebased segmentation approaches are not effective to segment the non-ideal iris images acquired in visible imaging as noise induced in unconstrained iris imaging is significantly high as compared to the imaging in the controlled environments.Reference [11] proposes an integro-differential constellation model for segmenting the iris and pupil regions. Similarly to the integro-differential operator, the algorithm is likely to fail if there is no significant contrast between pupillary and limbic boundaries. Reference [4] presents another promising approach for iris segmentation using neural network. The proposed approach exploits the color features for classification of sclera and iris pixels. However, we argue that the reported sclera and iris color features are not effectively discriminating sclera and iris pixels from other regions. As can be observed in Figure 1, most of the information contained in the hue component is missing, which will definitely affect the performance of the classifier. 


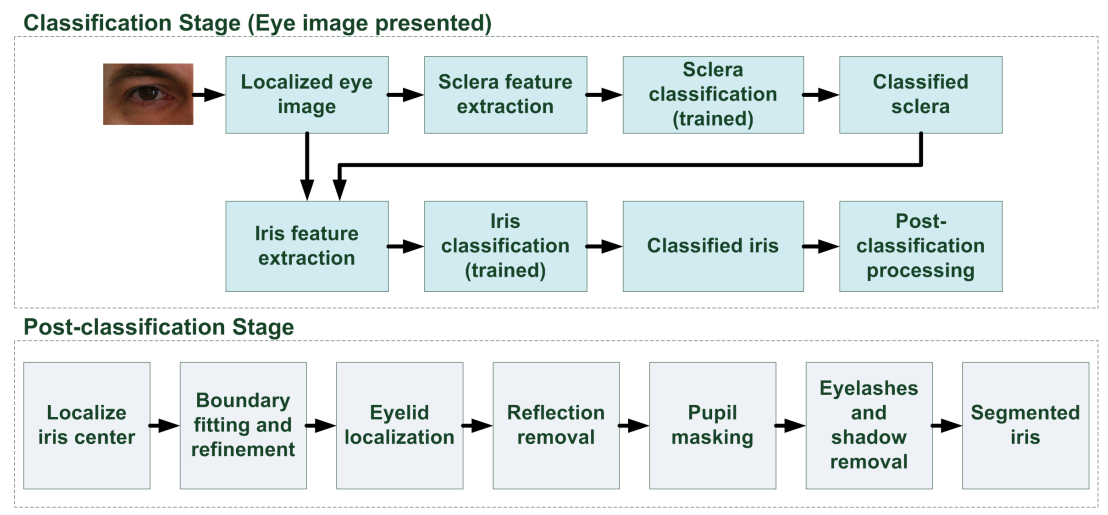

Figure 2. Block diagrams of proposed iris segmentation method.

\subsection{Our work}

Remote identification of human at-a-distance using iris feature requires development of completely automated and robust algorithm that can segment iris image pixels from distantly acquired facial images. Despite some initial efforts in the segmentation of such visible iris images from localized eye regions, the segmentation accuracy of such methods is quite limited. This paper focuses on such problem of iris image segmentation and develops a completely automated approach for iris segmentation from face image at-a-distance. The proposed approach simultaneously exploits two set of the features for sclera and iris classification. Iris features are extracted by exploiting localized Zernike moments [12] while sclera features are extracted by using discriminant color features. The experimental results from the proposed approach using neural network have achieved $42.4 \%$ improvement in segmentation accuracy on UBIRIS.v2 as compared to previous approach [4]. In addition, this paper also presents computationally simpler and fast alternative using SVM (support vector machine) classifier which has shown to offer $36.8 \%$ improvement in the average segmentation accuracy on UBIRIS.v2 over the approach in [4]. This paper has also detailed a robust approach for post-processing of classified iris image pixel (Section 2.3), which has been missing previously in [4]. The proposed scheme for such post-classification has been shown to be highly effective in reducing the iris segmentation errors due to the limitation in the classification stage.

The remainder of this paper is organized as follows. In Section 2, the proposed segmentation approach is detailed. The experiments and performance evaluation are presented in Section 3. Finally, the paper is concluded in Section 4.

\section{Iris segmentation for visible images}

The iris segmentation approach in this work is motivated by [4] which adopts a pixel-based strategy for the classification. Figure 2 shows the block diagram for iris segmentation when the acquired close-up eye image is presented, for example, images in UBIRIS.v1 [6] and UBIRIS.v2 databases. The proposed segmentation method can be divided into two processes: 1) classification and 2) post-classification. The classification process is mainly focus on coarse localizing of iris region while the post-classification process is to further refine the coarse segmentation results produced in the previous stage. In the classification stage, two classifiers are trained to detect the sclera and the iris regions. Both the sclera and iris features are extracted by exploiting discriminant color features and localized Zernike moments. The extracted features are used to train the neural network and SVM classifiers which will be used to classify image pixels into sclera and iris regions. The localized iris region is further refined in the post-classification stage (Section 2.3), which can greatly reduce the classification error due to the limitation of the classifiers.

\subsection{Feature extraction}

\subsubsection{Sclera features}

The sclera features are a 22-dimensional vector defined as follows

$\left\{x_{1}, x_{2}, S_{(\mathbf{0 , 2 , \mathbf { 4 }})}^{\mu \sigma}, n b_{(\mathbf{0 , 2 , \mathbf { 4 } )}}^{\mu \sigma}, d_{c r-c b(\mathbf{0 , 2 , \mathbf { 4 } )}}^{\mu \sigma}, d_{r g b}\left(x_{1}, x_{2}\right), \mu_{r g b}\left(x_{1}, x_{2}\right)\right\}$

where $S, n b, d_{c r-c b}, d_{r g b}$ and $\mu_{c}$ denote the saturation, normalized blue, difference of chroma red $(\mathrm{cr})$ and chroma blue $(c b)$, and mean of RGB, respectively. The coordinates $\left(x_{1}, x_{2}\right)$ indicate the spatial variables of the image. The subscripts in bold indicate the radii of the local window being processed centered at $\left(x_{1}, x_{2}\right)$. The superscripts indicate the mean and the standard deviation of a local window with respect to the radii are computed. Meanwhile, the $S$ and $n b$ components are subject to be preprocessed by subtracting the respective mean values. The $d_{c r-c b}, d_{r g b}$ and $\mu_{c}$ are defined as follows

$$
d_{c r-c b}\left(x_{1}, x_{2}\right)=c r\left(x_{1}, x_{2}\right)-c b\left(x_{1}, x_{2}\right)
$$




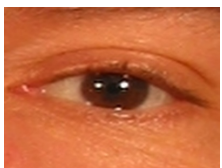

(a)

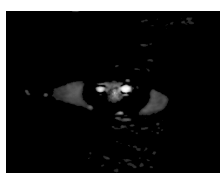

(b)

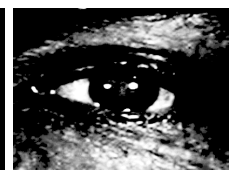

(c)

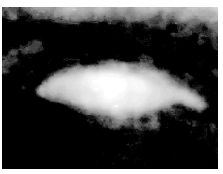

(d)

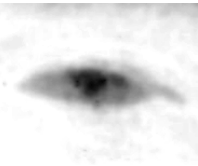

(e)

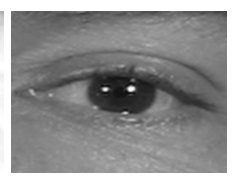

(f)

Figure 3. Proposed sclera features (a) original image, (b) saturation, (c) normalized blue, (d) difference of $c r c b$, (e) difference of $R G B$, (f) mean of $R G B$.

$$
\begin{gathered}
d_{r g b}\left(x_{1}, x_{2}\right)=2 I_{r}\left(x_{1}, x_{2}\right)-I_{g}\left(x_{1}, x_{2}\right)-I_{b}\left(x_{1}, x_{2}\right) \\
\mu_{r g b}\left(x_{1}, x_{2}\right)=1 / N \sum_{c \in\{r, g, b\}} I_{c}\left(x_{1}, x_{2}\right)
\end{gathered}
$$

where $N$ is the total number of color channels in image $I$. Figure 4 provides visual illustration of the proposed color features that are identified for sclera representation. The proposed 22-dimensional features are extracted from different color components which are observed empirically. The features derived from each of the color components serve to complement each other in order to enhance the discrimination between sclera and other regions. Sclera classification provides an important cue to coarsely estimate the location of the iris. Therefore, the iris features employed in our scheme also incorporate the classified sclera features, as detailed in the next subsection.

\subsubsection{Iris features}

Iris features are extracted by exploiting the localized Zernike moments and the classified sclera from the previous stage. Such features are defined as a 9-dimensional vector as follows

$$
\left\{x_{1}, x_{2}, I\left(x_{1}, x_{2}\right), Z_{60}^{2,5}(I), p_{w, e, n, s}\left(x_{1}, x_{2}\right)\right\}
$$

where $I$ is a grayscale image which representing only the red channel of the input color image [11]. The $Z$ is a function of $I$ centered at $\left(x_{1}, x_{2}\right)$ with respect to the radii indicated as superscript. The subscript of $Z$ denotes the order and the repetition of the Zernike moments, which is given as follows

$$
Z_{m n}=\frac{m+1}{\pi} \sum_{x_{1}} \sum_{x_{2}} f\left(x_{1}, x_{2}\right)\left[V_{m n}\left(x_{1}, x_{2}\right)\right]^{*} d x_{1} d x_{2}
$$

where $\left(x_{1}^{2}+x_{2}^{2} \leqslant 1\right)$. The subscript $m \in \mathbb{N}$ and $n \in \mathbb{Z}$ indicate the order and angular dependence of the Zernike moments. The $m-|n|$ must always be even and $|n| \leqslant m$. Function $f\left(x_{1}, x_{2}\right)$ is the sub-image/local region being processed and $V_{m n}$ is the Zernike polynomial [12]. The '*' denotes the complex conjugate of the function. The image $f$ to be processed must be first mapped to the unit circle expressed in polar coordinates. Besides using the Zernike moments, the classified sclera features which are the intermediate output from the previous stage have been incorporated as part of the unified iris features. The classified sclera is used to produce four proportion of sclera maps with respect to north $(n)$, south $(s)$, east $(e)$ and west $(w)$ directions. The proportions of sclera maps are responsible to delimit the iris region by considering the chosen four directions [4].

\subsection{Classification}

The classification of sclera and iris features using two types of classifiers is evaluated in our experiments. The first type of classifier is trained with feed forward network (FFN) classifier and the other type is SVM based classifier [13]. Both kinds of the classifiers are trained with the same training dataset except the fact that SVM is trained with least training samples ${ }^{1}$. The training configurations for both FFN-based and SVM-based classifiers are summarized in Table 1 and Table 2, respectively. The training samples (pixels) are randomly extracted from the training dataset (see Tables for the exact figures) and are evenly distributed among the positive and negative samples. To train the FFN classifiers, back propagation algorithm with Fletcher-Reeves learning method has been adopted. The FFN consists of three layers: input $(i)$, hidden $(h)$ and output (o) layers and is denoted as $N_{i}-N_{h}-N_{o}$, where $N_{\beta}$ indicates the number of neurons at layer $\beta \in\{i, h, o\}$. For FNN classifier, the same training approach is adopted. However, different kernel functions namely RBF (radial basis function) and linear are used to train the sclera and iris SVM classifiers, respectively. The kernel function are chosen based on the preliminary results obtained using the validation dataset.

\subsection{Post-classification stage}

\subsubsection{Iris center localization}

We have developed a simple and yet effective approach to automatically locate the center of iris from the circumcenter of a triangle formed by three control points. The three control points are extracted from a combined edge map, which is a summation result of two edge maps generated from the the classified iris mask and the corresponding grayscale ROI. In this work, the three control points are chosen by considering the edge points in horizontal and vertical directions from the center ${ }^{2}$ (see Figure 4 ). The proposed method

\footnotetext{
${ }^{1}$ The training dataset for SVM classifiers is the subset of training samples employed for $\mathrm{NN}$ classifiers.

${ }^{2}$ Theoretically, any three points on the edge map can be used.
} 


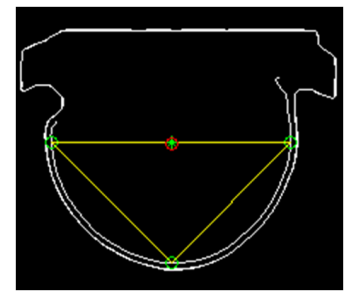

Figure 4. Iterative automatically iris center localization using three control points.

attempts to approximate the exact iris center which will be used in the subsequent processing, for example, boundary fitting, eyelid localization and eyelashes and shadow removal. Therefore, the proposed method provides a simple way to approximate the iris center since our focus is find a generally good reference point to be used in the subsequent post-classification steps ${ }^{3}$. One should note that the combined edge maps may contain short edge lines, which may affect the iris center localization performance. Thus, it is essential to remove the relative short edges before finding the iris center. The initial iris center $\left(C_{x_{1}}, C_{x_{2}}\right)$ is obtained by taking the mean $\left(\mathbf{x}_{1}, \mathbf{x}_{2}\right)$ of the segmented binary mask.

After obtaining the iris center $\left(C_{x_{1}}, C_{x_{2}}\right)$, three control points are extracted from the combined edge map which virtually form a triangle. Circumcenter of this triangle is then calculated and the $\left(C_{x_{1}}, C_{x_{2}}\right)$ is updated with this value. The process is iterated until the $\left(C_{x_{1}}, C_{x_{2}}\right)$ is converged or the predefined stopping criteria is met. The converged circumcenter provides the clues of the iris center $\left(C_{x_{1}}^{\prime}, C_{x_{2}}^{\prime}\right)$ and the radius $r$ from the center to the boundary. In figure 4, the '*' indicates the initial iris center $\left(C_{x_{1}}, C_{x_{2}}\right)$, the green 'o' indicates the three control points and the red 'o' denotes the calculated circumcenter.

\subsubsection{Boundary refinement}

The boundary of the classified iris is refined by using the polynomial curve fitting of degree 3 . The combined edge map obtained in previous section is used again by transforming it to the polar coordinate system with respect to the $\left(C_{x_{1}}^{\prime}, C_{x_{2}}^{\prime}\right)$ and $r$. In order to mitigate the influence of the noisy points to the system, we adopt the strategy by selecting only one edge point per column [14] as the chosen control points for the boundary fitting.

\subsubsection{Eyelid localization}

The eyelid localization steps use estimated iris centers $\left(C_{x_{1}}^{\prime}, C_{x_{2}}^{\prime}\right)$ as reference and partition the localized iris into two regions, i.e., lower and upper eyelids, which are delimited by radius $r$. The edge points are extracted for each of these regions by firstly applying Canny edge detector on the

\footnotetext{
${ }^{3}$ The true iris center can be obtained after the boundary refinement step.
}

Table 1. Training configurations for FFN-based classifiers.

\begin{tabular}{|l|c|c|}
\hline & Sclera & Iris \\
\hline Total \# of train images & 30 & 35 \\
\# of features per pixel & 22 & 9 \\
\# of +/- samples & 35000 & 35000 \\
\# of layers \& neurons & $22-28-1$ & $9-14-1$ \\
Radii of windows & $0,2,4$ & 2,5 \\
Order of ZMs & - & 6 \\
Learning algorithm & Fletcher-Reeves & Fletcher-Reeves \\
\hline
\end{tabular}

Table 2. Training configurations for SVM-based classifiers.

\begin{tabular}{|l|c|c|}
\hline & Sclera & Iris \\
\hline Total \# of train images & 30 & 35 \\
\# of features per pixel & 22 & 9 \\
\# of +/- samples & 10000 & 5000 \\
Kernel & RBF & Linear \\
Radii of windows & $0,2,4$ & 2,5 \\
\hline
\end{tabular}

extracted ROI of the grayscale input image ${ }^{4}$ defined by the refined iris mask. Similarly, we apply the same strategy to extract one edge point per column for each upper and lower region to mitigate the effect of the possible outliers. We use the polynomial curve fitting with degree 2 to fit a parabolic curve for each upper and lower eyelid, as depicted in Figure 5. The area which has fallen outside the localized upper and lower eyelids is removed from the iris mask.

\subsubsection{Reflection removal}

Unlike the images acquired using NIR illumination, the reflection induced in VW iris images is often severe. Simple thresholding technique as usually applied on the NIR iris images by assuming high intensity values for reflection is tend to fail in VW iris images as the assumption may not hold due to a number of factors induced in the unconstrained imaging environments, for instance, ambient light. Figure 6(a) and (b) show a typical example of an iris image contaminated with the reflection noise and its corresponding iris mask, respectively. By considering that the intensity distribution of a valid iris region is relatively close and the reflection is consuming only a small portion, we have transformed the intensity histogram $H$ using the following function

$$
G(i)=e^{\frac{\left(H(i)-H_{\mu}\right)^{2}}{2 H_{\sigma}^{2}}}
$$

where $i$ denotes the intensity level, $H_{\mu}$ and $H_{\sigma}$ denote the mean and the standard deviation of the histogram $H$. As can be seen in Figure 6(c), the transformed histogram $G$ has relatively flat tails at both ends of the plot. In order to obtain an adaptive threshold to mask out the reflection region, the

\footnotetext{
${ }^{4}$ The red channel of a color input image is used in all experiments.
} 


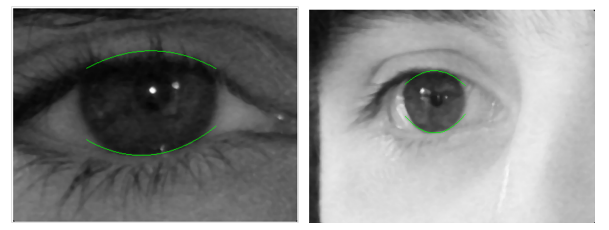

Figure 5. Upper and lower eyelid localization using parabolic curve fitting.

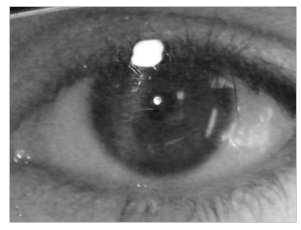

(a)

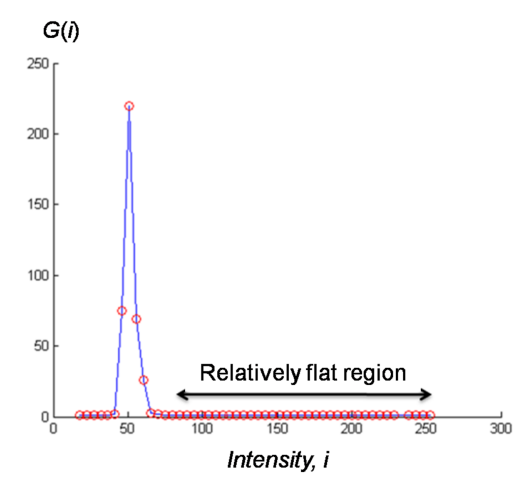

(c)

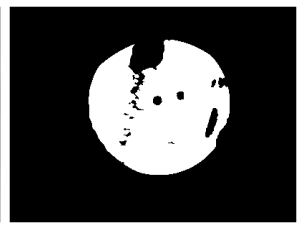

(b) input image, (b) iris mask with Figure 6. Reflection removal (a) input image, (b) iris mask with
reflection removed, (c) transformed histogram of iris region (a).

differences of the adjacent bins of $G$ are compared starting from the right-tail. The adaptive threshold is obtained if the absolute difference of $G$ is greater than a predefined threshold $\tau$, i.e. $|G(i)-G(i-1)|>\tau$. The predefined threshold $\tau$ is empirically set to 0.06 in all experiments. Therefore for the absolute differences of $G$ below $\tau$, the corresponding bin is treated as belonging to the flat region.

\subsubsection{Pupil masking}

The pupil in VW iris images is often more challenging to localize as the contrast level between iris and pupil regions are not as high compared to those acquired using NIR illumination. Therefore, we again make use of the intensity histogram $H$ of the extracted iris region to calculate adaptive threshold to mask the pupil region, i.e. $T=$ $\alpha \times\left(\varphi(\max (H))+\varphi_{0}\right)$, where function $\max$ returns the index of the maximum frequency of $H$. The function $\varphi$ returns the independent variable of $H$ given an index and $\varphi_{0}$ indicates the first independent variable of $H$. The $\alpha$ can be considered as a weight factor which determines the adaptive threshold $T$ ( $\alpha$ is set to 0.4 in all experiments).

\subsubsection{Eyelashes and shadow removal}

Our eyelash and shadow removal approach is largely based on [14] which also exploits the difference in intensity distribution of pixels from the eyelashes and shadow (ES) regions. The localized iris is separated into two regions namely ES region and IR (iris) region. The ES region is defined as the area from the upper eyelid and delimited by a distance, dist, given as $($ dist $=r \times 0.3)$ where $r$ is the radius of the iris obtained previously. The histograms of these regions are constructed and subject to the transformation using (7). The middle point between the peaks of the two transformed histograms is used as the threshold to mask the eyelashes and shadow in ES region.

\section{Experiments and results}

Repeatability and reproducibility of experiment results are one of the important issues in biometrics as researchers often find it is difficult to reproduce the published experimental results. One of the key reasons, especially in the context of visible imaging iris recognition, is the absence of details on the selection of training/test samples. In order to ascertain the performance of the proposed segmentation approach, UBIRIS.v2 as being one of the publicly available databases acquired using visible imaging in unconstrained imaging environments was utilized. In this work, the subset of the UBIRIS.v2 which consists of 1000 iris images released for NICE:II [15] competition was used in the experiments. The noise free iris masks have been made available along with the release of this subset of the UBIRIS.v2. Therefore, we have manually generated the ground truth from 1000 images for the performance evaluation. The first 96 iris images from the UBIRIS.v2 subset is chosen as training and validation datasets, as 41 images for training and 55 images for validation. The remaining 904 images are used as independent test data to ascertain the performance.

The evaluation protocol used in NICE.I competition [16] has been adopted to evaluate the performance of the proposed segmentation approach. The segmentation error $E$ is a measurement of total disagreeing pixels between the segmented mask and the corresponding group truth sclera/iris mask. The experimental results obtained by our proposed method are summarized in Table 3 as well as the comparison with one state-of-the-art method [4]. Our proposed method has gained the improvement of $42.4 \%$ for UBIRIS.v2, as compared to the method in [4]. Figure 7(a) shows some samples segmentation results of our proposed method applied on UBIRIS.v2 database.

\section{Conclusions}

This paper has developed a new approach for completely automated iris segmentation from the images acquired at-adistance using visible illumination. Unlike the traditional 
Table 3. Performance evaluation on UBIRIS.v2 dataset.

\begin{tabular}{|l|c|c|}
\hline \multirow{2}{*}{} & \multicolumn{2}{|c|}{ Error, $E(\%)$} \\
\cline { 2 - 3 } & Sclera & Iris \\
\hline Proposed method (NN) & 4.51 & 2.16 \\
Proposed method (SVM) & 3.26 & 2.37 \\
Previous method [4] & 4.99 & 3.75 \\
\hline
\end{tabular}

edge-based segmentation approaches, the proposed technique exploits the discriminative color features and localized Zernike moments to perform pixel-based classification. The pixel-based strategy relaxes the requirements to have a priori knowledge about the capturing ranges between subjects and acquisition devices. This paper has also detailed the selection of training/test data for all experiments to ensure repeatability of the proposed method. Such needed details have been missing to ensure repeatability in previous methods. The rigorous experimental results presented in this paper have shown improvement of $42.4 \%$ in the average segmentation errors for UBIRIS.v2 as compared to previous approach. The experimental results presented in this paper have illustrated the robustness of the proposed segmentation approach. The best of the segmentation results are obtained using FFN classifier. The FFN classifiers are known to suffer from the problems of local minima and require rigorous training to ensure better performance. Any alternative classifiers that can provide better or similar performance as using FFN classifier is desirable. Therefore, SVM can be a good alternative to the FFN. The performance of the SVM can be justified from the experimental results for sclera classification. The performance achieved using FFN is marginally superior than SVM and this could be possibly due to the lack of adequate training samples (see Table 2).

The experimental results presented in this paper are very promising and also bring several issues to be addressed in further work. It is likely that the demand and applications of the VW iris recognition will significantly increase in near future, mainly due to the availability of low cost and high resolution imaging sensors in mobile and surveillance cameras. The VW iris segmentation approach developed in this work has been rigorously evaluated on publicly available VW database. We are currently experimenting the segmentation approach on the FRGC database [17] as well as extending our work on iris images acquired using NIR illumination. Figure 7(b) shows some preliminary segmentation results obtained using the proposed algorithm applied on the FRGC dataset.

\section{Acknowledgement}

This work is partially supported by the internal competitive research grant from The Hong Kong Polytechnic University (2009-2010), grant no. PJ70.

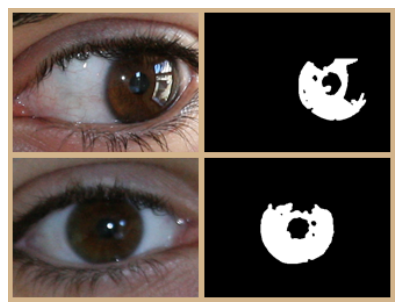

(a)

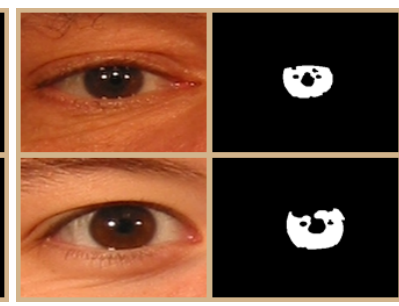

(b)
Figure 7. Sample segmentation results of proposed approach applied on the databases (a) UBIRIS.v2, (b) FRGC.

\section{References}

[1] K. Bowyer, K. Hollingsworth, and P. Flynn, "Image understanding for iris biometrics: A survey," Image Vision Comput., vol. 110, no. 2, pp. 281-307, 2008. 9

[2] J. Daugman, "How iris recognition works," IEEE Trans. Circuits Syst. Video Technol., vol. 14, no. 1, pp. 21-30, 2004. 9

[3] H. Proenca, S. Filipe, R. Santos, J. Oliveira, and L. Alexandre, "The UBIRIS.v2: A database of visible wavelength images captured onthe-move and at-a-distance," IEEE Trans. Pattern Anal. Mach. Intell., vol. 32, no. 8, pp. 1529-1535, 2010. 9

[4] H. Proenca, "Iris recognition: On the segmentation of degraded images acquired in the visible Wavelength," IEEE Trans. Pattern Anal. Mach. Intell., vol. 32, no. 8, pp. 1502-1516, 2010. 9, 10, 11, 13, 14

[5] N. Kourkoumelis and M. Tzaphlidou, "Medical safety issues concerning the use of incoherent infrared light in biometrics," in Ethics and Policy of Biometrics (A. Kumar and D. Zhang, eds.), vol. 6005 of LNCS, pp. 121-126, Springer Berlin / Heidelberg, 2010. 9

[6] H. Proenca and L. Alexandre, "UBIRIS: A noisy iris image database," in Proceed. of ICIAP 2005 - Intern. Confer. on Image Analysis and Processing, vol. 1, 2005. 9, 10

[7] J. Daugman, "New methods in iris recognition," IEEE Trans. Syst. Man Cybern. Part B Cybern., vol. 37, no. 5, pp. 1167-1175, 2007. 9

[8] S. Schuckers, N. Schmid, A. Abhyankar, V. Dorairaj, C. Boyce, and L. Hornak, "On techniques for angle compensation in nonideal iris recognition,' IEEE Trans. Syst. Man Cybern. Part B Cybern., vol. 37, no. 5, pp. 1176-1190, 2007. 9

[9] K. Miyazawa, K. Ito, T. Aoki, K. Kobayashi, and H. Nakajima, "An effective approach for iris recognition using phase-based image matching," IEEE Trans. Pattern Anal. Mach. Intell., vol. 30, no. 10, pp. 1741-1756, 2008. 9

[10] A. Kumar and A. Passi, "Comparison and combination of iris matchers for reliable personal authentication," Pattern Recognit., vol. 43, no. 3, pp. 1016-1026, 2010. 9

[11] T. Tan, Z. He, and Z. Sun, "Efficient and robust segmentation of noisy iris images for non-cooperative iris recognition," Image Vision Comput., vol. 28, no. 2, pp. 223-230, 2010. 9, 11

[12] J. Shutler, "Complex Zernike moments," 8 2002. http: //homepages.inf.ed.ac.uk/rbf/CVonline/LOCAL_ COPIES/SHUTLER3/node11.html. 10, 11

[13] C.-C. Chang and C.-J. Lin, "LIBSVM : a library for support vector machines," 2001. http://www.csie.ntu.edu.tw/ cjlin/libsvm. 11

[14] Z. He, T. Tan, Z. Sun, and X. Qiu, "Toward accurate and fast iris segmentation for iris biometrics," IEEE Trans. Pattern Anal. Mach. Intell., vol. 31, no. 9, pp. 1670-1684, 2009. 12, 13

[15] NICE:II - Noisy Iris Challenge Evaluation, Part II. http:// nice2.di.ubi.pt/. 13

[16] NICE.I - Noisy Iris Challenge Evaluation, Part I. http://nice1. di.ubi.pt/index.html. 13

[17] Face Recognition Grand Challenge - Overview. http://www. frvt.org/FRGC/. 14 